\title{
Высокочастотная проводимость неупорядоченных полупроводников в области перехода от линейной к квадратичной частотной зависимости
}

\author{
(C) М.А. Ормонт, И.П. Звягин \\ Московский государственный университет им. М.В. Ломоносова (физический фракультет), \\ 119991 Москва, Россия \\ E-mail: ormont@phys.msu.ru
}

Поступила в Редакцию 18 марта 2019 г.

В окончательной редакции 2 сентября 2019 г.

Принята к публикации 5 сентября 2019 г.

Рассмотрены особенности высокочастотной проводимости неупорядоченных полупроводников, связанные с прыжковым транспортом электронов по примесной зоне. В рамках парного приближения проведен расчет частотной зависимости вещественной части низкотемпературной бесфононной проводимости в области перехода (кроссовера) от линейной к квадратичной частотной зависимости проводимости. Показано, что кроссовер в терагерцовом диапазоне частот связан с переходом от режима проводимости с переменной длиной прыжка к режиму проводимости с постоянной длиной прыжка с ростом частоты.

Ключевые слова: высокочастотная прыжковая проводимость, универсальность частотной зависимости проводимости, неупорядоченные полупроводники.

DOI: 10.21883/FTP.2020.01.48766.9106

\section{1. Введение}

Для многих неупорядоченных материалов (аморфные и легированные полупроводники, полупроводниковые стекла, проводящие полимеры, гранулированные проводники и т.п.) частотная зависимость вещественной части проводимости в широкой области частот хорошо описывается степенным законом $[1,2]$

$$
\sigma_{1}(\omega)=C \omega^{s}
$$

где $C, s-$ постоянные (как правило, $0<s<1$ ). Возрастающая степенная частотная зависимость (1) указывает на прыжковый характер транспорта, однако универсальность зависимости (1) затрудняет получение информации о конкретных особенностях механизма переноса из вида частотной зависимости проводимости. По этой причине важную роль приобретают исследования отклонений частотной зависимости проводимости от универсальности $(s \approx 1)$ и установление их связи с особенностями механизма переноса и со структурными особенностями материала.

В случае прыжкового переноса по локализованным состояниям примесной зоны слабо легированных компенсированных полупроводников теория предсказывает частотные зависимости вещественной части комплексной проводимости вида $\sigma_{1}(\omega) \sim r_{\omega}^{n} \omega^{m} \sim \omega^{m} \ln ^{n}\left(\omega_{c, \mathrm{ph}} / \omega\right)$ (где $n, m$ - целые числа, $r_{\omega}-$ характерная длина прыжка на частоте $\omega, \omega_{\mathrm{ph}}-$ характерная фононная частота, представляющая собой частоту попыток перехода электрона при релаксационной прыжковой проводимости, $\omega_{c}$ - частота попыток перехода электрона при резонансной проводимости) [1]. Функции вида $\omega^{m} \ln ^{n}\left(\omega_{c, \mathrm{ph}} / \omega\right)$, фигурирующие в выражениях для про-

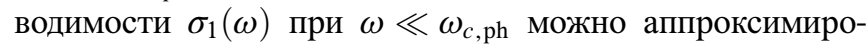
вать степенным законом $A \omega^{s}$ с показателем степени

$$
s=\frac{d \ln (\sigma(\omega))}{d \ln \omega}=m-\frac{n}{\ln \left(\omega_{c, \mathrm{ph}} / \omega\right)} ;
$$

при этом показатель степени $s$ уменьшается с ростом частоты $\omega$.

Степенная частотная зависимость (1) обычно связывается с прыжками электронов по локализованным состояниям с участием фононов (релаксационная проводимость) [3-5]. Частотная зависимость вещественной части проводимости, близкая к линейной, получается при низких частотах и в теории низкотемпературной бесфононной (резонансной) прыжковой проводимости при учете кулоновских корреляций [6]. Теория бесфононной проводимости предсказывает переход (кроссовер) от линейной частотной зависимости $(s \approx 1[6])$ к зависимости, близкой к квадратичной $(s \approx 2$ [7]), в области частот порядка $\omega_{c r}$, при которых $\hbar \omega$ становится порядка энергии кулоновского взаимодействия между электронами внутри резонансных пар $U\left(r_{\omega}\right)$. В области более низких частот вещественная часть проводимости определяется фононным механизмом, а с ростом частоты бесфононный вклад в проводимость начинает преобладать над релаксационным. Низкотемпературные измерения (при $T \sim 1 \mathrm{~K})$ частотной зависимости вещественной части проводимости $\sigma_{1}(\omega)$ в легированном кремнии $(\mathrm{Si}: \mathrm{P})$ на изоляторной стороне перехода металл-диэлектрик показали, что $\omega_{c r} \sim 1$ ТГц $[8,9]$.

Частотная зависимость характерной длины прыжка $r_{\omega}$ в режиме резонансной проводимости связана с гибридизацией электронных состояний. Согласно тео- 
рии $[6,7]$, из-за гибридизации волновых функций изолированной пары центров и соответствующего ей отталкивания уровней наибольший вклад в бесфононную проводимость вносят пары центров, для которых межцентровые расстояния $r_{\lambda \lambda^{\prime}}$ удовлетворяют неравенствам $r_{\omega} \leq r_{\lambda \lambda^{\prime}} \leq r_{\omega}+a$, где $r_{\omega}=a \ln \left(2 I_{0} / \hbar \omega\right), \quad I_{0} \approx e^{2} / \kappa a$, $a$ - радиус локализации состояний, $\kappa$ - диэлектрическая проницаемость материала; при $r_{\lambda \lambda^{\prime}}<r_{\omega}$ отталкивание уровней за счет гибридизации состояний становится бо́льшим $\hbar \omega$, так что резонансные переходы невозможны. В парном приближении характерная длина прыжка $r_{\omega}$ на частоте $\omega$ определяется из условия $\hbar \omega=2 I_{\lambda \lambda^{\prime}}\left(r_{\omega}\right)$, где $I_{\lambda \lambda^{\prime}}=I_{0} \exp \left(-r_{\lambda \lambda^{\prime}} / a\right)-$ резонансный интеграл, а $\lambda$ - номер центра. Из теории бесфононной проводимости систем водородоподобных центров локализации следует, что предэкспоненциальный множитель $I_{0}$ резонансного интеграла $I_{\lambda \lambda^{\prime}}$ служит основным параметром рассматриваемой задачи, определяющим критическую частоту $\omega_{c}=2 I_{0} / \hbar$. Для систем с водородоподобными примесными центрами ( $\mathrm{Si}: \mathrm{P})$ характерное значение критической частоты составляет $v_{c}=\omega_{c} / 2 \pi \sim 10$ ТГц.

С ростом частоты $\omega$ характерная длина прыжка $r_{\omega}$ уменьшается. Проведенное в работе [10] рассмотрение показало, что в силу частотной зависимости характерной длины прыжка $r_{\omega}$ частотная зависимость $\sigma_{1}(\omega)$ может быть немонотонной, причем вплоть до частоты $\omega_{m}$, отвечающей максимуму $\sigma_{1}(\omega)$, кулоновское взаимодействие между электронами „активных“ (резонансных) пар играет основную роль $\hbar \omega<U\left(r_{\omega}\right)=e^{2} / \kappa r_{\omega}$ и частотная зависимость $\sigma_{1}(\omega)$ остается близкой к линейной. При типичных для мелких примесей значениях параметра $A=\frac{e^{2}}{\kappa a \hbar \omega_{c}} \approx 0.5$ частотные зависимости проводимости $\sigma_{1}(\omega)$ в режиме с переменной длиной прыжка $r_{\omega}$ не описывают наблюдаемый переход частотной зависимости проводимости от почти линейной к квадратичной с ростом частоты. Существенно более плавный переход, чем наблюдается на опыте, от линейной к квадратичной частотной зависимости проводимости $\sigma_{1}(\omega)$ в режиме с переменной длиной прыжка можно объяснить лишь при нетипичных для мелких примесей значениях параметра $A<10^{-5}$.

Немонотонность частотной зависимости вещественной части проводимости $\sigma_{1}(\omega)$ определяется двумя конкурирующими тенденциями. Рост $\sigma_{1}(\omega)$ с частотой связан с увеличением энергии поглощаемого кванта $\hbar \omega$ и с увеличением числа исходных центров $\sim \rho_{0}\left(\hbar \omega+U\left(r_{\omega}\right)\right)$. Здесь $\rho_{0}-$ одночастичная плотность состояний, считаемая постоянной. Уменьшение $\sigma_{1}(\omega)$ с частотой определяется уменьшением квадрата изменения дипольного момента системы $\left(e r_{\omega}\right)^{2}$ и уменьшением числа конечных центров $\sim \rho_{0} S_{\omega} a$, где $S_{\omega} a=4 \pi r_{\omega}^{2} a-$ объем сферического слоя, в который происходят переходы. Однако предсказанная теорией немонотонность частотной зависимости бесфононной проводимости в легированных компенсированных полупроводниках экспе- риментально не была обнаружена [8,9]. Таким образом, в рамках стандартных представлений о переменной длине прыжка $r_{\omega}$, зависящей от частоты, теория резонансной проводимости не описывает поведение проводимости в области перехода от линейной к квадратичной частотной зависимости [10].

Наблюдаемый переход от линейной к квадратичной частотной зависимости проводимости может быть связан с переходом к режиму проводимости с постоянной длиной прыжка, когда основной вклад в проводимость вносят электронные переходы внутри пар с оптимальными межцентровыми расстояниями $r_{\text {opt }}$, слабо зависящими от частоты [11]. В этом случае оптимальная длина прыжка $r_{\text {opt }}$ перестает зависеть от частоты за счет подавления гибридизации электронных состояний: с ростом частоты основной вклад в проводимость начинают вносить пары центров, в которых электрон не успевает гибридизоваться. Для заданной пары центров $\lambda, \lambda^{\prime}$ гибридизация характеризуется частотой Раби: $\omega_{\mathrm{R}}=2 I\left(r_{\lambda \lambda^{\prime}}\right) / \hbar$, при этом для пар с межцентровыми расстояниями $r_{\lambda \lambda^{\prime}} \geq r_{\omega}$ частота Раби меньше частоты поля, т.е. $\omega>\omega_{\mathrm{R}}$, и в этом случае эффекты, связанные с гибридизацией локализованных состояний, становятся несущественны. Существование оптимального расстояния $r_{\text {opt }}$ между центрами в парах обусловлено тем, что с уменьшением расстояния между центрами в паре уменьшается и изменение дипольного момента системы при электронном переходе, а с увеличением расстояния между центрами происходит экспоненциальное уменьшение перекрытия волновых функций состояний, отвечающих центрам локализации. Соответственно при высоких частотах, когда эффекты гибридизации несущественны и оптимальная длина прыжка $r_{\text {opt }}$ не зависит от частоты, выражение для вещественной части проводимости принимает вид $[11]$

$$
\sigma_{1}(\omega)=\frac{\pi^{2}}{3} C_{1} e^{2} \rho_{0}^{2} a^{5} \omega\left(\hbar \omega+\frac{e^{2}}{\kappa r_{\mathrm{opt}}}\right),
$$

где $r_{\text {opt }} \approx 3 a-$ оптимальная длина прыжка, определяющаяся параметрами системы, $C_{1}-$ численный коэффициент. Не зависящая от частоты характерная длина прыжка $r_{\text {opt }}$ обусловливает монотонный характер частотной зависимости вещественной части проводимости. Согласно (2), переход от линейной частотной зависимости вещественной части проводимости к квадратичной с повышением частоты происходит при частоте $\omega \sim \omega_{c r}$, где частота кроссовера $\omega_{c r}$ определяется равенством $\hbar \omega_{c r}=U\left(r_{\text {opt }}\right)=e^{2} / \kappa r_{\text {opt}}$, т. е. $\omega_{c r} \approx 0.1 \omega_{c}$, где $\omega_{c}=2 I_{0} / \hbar$; при $a \approx 30 \AA(\mathrm{Si}: \mathrm{P})$ имеем $v_{c r}=\omega_{c r} / 2 \pi \approx 1$ ТГц.

$\mathrm{C}$ ростом частоты характерная длина прыжка $r_{\omega}$ уменьшается, и в области высоких частот происходит переход к режиму проводимости с постоянной длиной прыжка (2), когда основной вклад в проводимость вносят электронные переходы внутри пар с оптимальными 
межцентровыми расстояниями $r_{\text {opt }}$, слабо зависящими от частоты. Согласно [11], переход к режиму проводимости с постоянной длиной прыжка $r_{\text {орt }}$ происходит при $r_{\omega} \approx r_{\text {opt }}$, т. е. при частоте $\omega_{\text {opt }} \approx 0.05 \omega_{c}$.

В работах $[11,12]$ был проведен анализ частотных зависимостей проводимости в режимах проводимости с переменной и постоянной (не зависящей от частоты) длиной прыжка; при этом частотная зависимость проводимости в области кроссовера получалась экстраполяцией модели с переменной длиной прыжка на область квадратичной частотной зависимости в режиме проводимости с постоянной длиной прыжка. Цель настоящей работы состояла в непосредственном расчете в рамках парного приближения частотной зависимости низкотемпературной бесфононной проводимости в области перехода от линейной к квадратичной частотной зависимости проводимости. Такой расчет позволяет показать, что кроссовер частотной зависимости проводимости непосредственно связан с переходом от режима проводимости с переменной длиной прыжка к режиму проводимости с постоянной длиной прыжка с ростом частоты в окрестности кроссовера.

\section{2. Бесфононная проводимость в области перехода от линейной к квадратичной частотной зависимости}

В слабых электрических полях в парном приближении выражение для бесфононной проводимости имеет вид [13]

$$
\begin{aligned}
\sigma_{1}^{\mathrm{res}}(\omega) & =\frac{\pi e^{2} \omega}{V_{0}} \sum_{\substack{\left\{\lambda, \lambda^{\prime}\right\} \\
\lambda \neq \lambda^{\prime}}}\left|\left\langle\psi_{\lambda \lambda^{\prime}}^{-}|(\mathbf{n}, \mathbf{r})| \psi_{\lambda \lambda^{\prime}}^{+}\right\rangle\right|^{2} \\
& \times\left(n_{F}\left(\varepsilon_{\lambda \lambda^{\prime}}^{-}\right)-n_{F}\left(\varepsilon_{\lambda \lambda^{\prime}}^{+}\right)\right) \delta\left(\varepsilon_{\lambda \lambda^{\prime}}^{-}-\varepsilon_{\lambda \lambda^{\prime}}^{+}+\hbar \omega\right),
\end{aligned}
$$

где $\psi_{\lambda \lambda^{\prime}}^{ \pm}=C_{\lambda}^{ \pm} \psi_{\lambda}+C_{\lambda^{\prime}}^{ \pm} \psi_{\lambda^{\prime}}-$ гибридизованные волновые функции основных состояний $\psi_{\lambda}, \psi_{\lambda^{\prime}}$ электрона на изолированных центрах локализации $\lambda$ и $\lambda^{\prime}, \mathbf{n}-$ единичный вектор, параллельный внешнему электрическому полю, $n_{F}(\varepsilon)$ - среднее число заполнения состояния с энергией $\varepsilon, V_{0}$ - объем системы. Волновые функции $\psi_{\lambda \lambda^{\prime}}^{-}, \psi_{\lambda \lambda^{\prime}}^{+}$ отвечают нижнему $\varepsilon_{\lambda \lambda^{\prime}}^{-}$и верхнему $\varepsilon_{\lambda \lambda^{\prime}}^{+}$уровням энергии,

$$
\varepsilon_{\lambda \lambda^{\prime}}^{ \pm}=\frac{\varepsilon_{\lambda}^{0}+\varepsilon_{\lambda^{\prime}}^{0}}{2} \pm \frac{1}{2} \sqrt{\left(\varepsilon_{\lambda}^{0}-\varepsilon_{\lambda^{\prime}}^{0}\right)^{2}+4 I_{\lambda \lambda^{\prime}}^{2}},
$$

$\varepsilon_{\lambda}^{0}, \varepsilon_{\lambda^{\prime}}^{0}-$ затравочные энергии (без учета гибридизации), $\quad I_{\lambda \lambda^{\prime}}=\left\langle\psi_{\lambda}\left|\hat{U}_{\lambda}\right| \psi_{\lambda^{\prime}}\right\rangle-$ резонансный интеграл; $\hat{U}_{\lambda} \approx \hat{\tilde{U}}_{\lambda}+e \varphi\left(\mathbf{r}_{\lambda}\right)-$ потенциальная энергия локализованного электрона в точке расположения центра с номером $\lambda$, учитывающая кулоновский сдвиг e $\varepsilon\left(\mathbf{r}_{\lambda}\right)$, обусловленный другими заряженными центрами в точке $\mathbf{r}_{\lambda}$. При этом волновая функция основного состояния электрона, локализованного в окрестности центра, остается практически неизменной и близкой к волновой функции, соответствующей изолированному центру. Для гибридизованных волновых функций $\psi_{\lambda \lambda^{\prime}}^{-}, \psi_{\lambda \lambda^{\prime}}^{+}$основных состояний электрона на изолированных центрах локализации $\lambda$ и $\lambda^{\prime}$ имеем

$$
\begin{aligned}
& \psi_{\lambda \lambda^{\prime}}^{-}=\frac{1}{\sqrt{1+\frac{4 I_{\lambda \lambda^{\prime}}^{2}}{\left(\varepsilon_{\lambda}^{0}-\varepsilon_{\lambda^{\prime}}^{0}+\Gamma_{\left.\lambda \lambda^{\prime}\right)^{2}}\right.}}\left(-\frac{2 I_{\lambda \lambda^{\prime}}}{\left(\varepsilon_{\lambda}^{0}-\varepsilon_{\lambda^{\prime}}^{0}+\Gamma_{\lambda \lambda^{\prime}}\right)} \psi_{\lambda}+\psi_{\lambda^{\prime}}\right),} \\
& \psi_{\lambda \lambda^{\prime}}^{+}=\frac{1}{\sqrt{1+\frac{4 I_{\lambda \lambda^{\prime}}^{2}}{\left(\varepsilon_{\lambda}^{0}-\varepsilon_{\lambda^{\prime}}^{0}+\Gamma_{\lambda \lambda^{\prime}}\right)^{2}}}}\left(\psi_{\lambda}+\frac{2 I_{\lambda \lambda^{\prime}}}{\left(\varepsilon_{\lambda}^{0}-\varepsilon_{\lambda^{\prime}}^{0}+\Gamma_{\lambda \lambda^{\prime}}\right)} \psi_{\lambda^{\prime}}\right),
\end{aligned}
$$

где $\Gamma_{\lambda \lambda^{\prime}}=\sqrt{\left(\varepsilon_{\lambda}^{0}-\varepsilon_{\lambda^{\prime}}^{0}\right)^{2}+4 I_{\lambda \lambda^{\prime}}^{2}}$. Из выражений $(5 \mathrm{a})$, (5б) следует, что при $\varepsilon_{\lambda}^{0}=\varepsilon_{\lambda^{\prime}}^{0}$ волновые функции гибридизованных состояний $\psi_{\lambda \lambda^{\prime}}^{ \pm}$и соответствующие им энергии $\varepsilon_{\lambda \lambda^{\prime}}^{ \pm}$равны

$$
\begin{aligned}
& \psi_{\lambda \lambda^{\prime}}^{-}=\frac{1}{\sqrt{2}}\left(\psi_{\lambda}+\psi_{\lambda^{\prime}}\right), \\
& \psi_{\lambda \lambda^{\prime}}^{+}=\frac{1}{\sqrt{2}}\left(\psi_{\lambda}-\psi_{\lambda^{\prime}}\right),
\end{aligned}
$$

$\varepsilon_{\lambda \lambda^{\prime}}^{ \pm}=\varepsilon_{\lambda}^{0} \pm\left|I_{\lambda \lambda^{\prime}}\right|$, т. е. $\varepsilon_{\lambda \lambda^{\prime}}^{+}-\varepsilon_{\lambda \lambda^{\prime}}^{-}=2\left|I_{\lambda \lambda^{\prime}}\right|$. Соответственно в состояниях, отвечающих волновым функциям (6а), (6б), электрон с равной вероятностью может находиться в окрестности каждого из двух центров локализации $\lambda, \lambda^{\prime}$ в независимости от межцентрового расстояния. Качественно такое поведение носителя заряда сохраняется при малом разбросе энергий уровней, когда $\left|\varepsilon_{\lambda}^{0}-\varepsilon_{\lambda^{\prime}}^{0}\right| \ll 2\left|I_{\lambda \lambda^{\prime}}\right|$.

В противоположном случае из большого разброса энергий уровней, $\left|\varepsilon_{\lambda}^{0}-\varepsilon_{\lambda^{\prime}}^{0}\right| \gg 2\left|I_{\lambda \lambda^{\prime}}\right|$, из (5a), (5б) следует, что волновые функции $\psi_{\lambda \lambda^{\prime}}^{ \pm}$и соответствующие им энергии $\varepsilon_{\lambda \lambda^{\prime}}^{ \pm}$равны

$$
\begin{aligned}
& \psi_{\lambda \lambda^{\prime}}^{-} \approx \frac{\left|I_{\lambda \lambda^{\prime}}\right|}{\left(\varepsilon_{\lambda}^{0}-\varepsilon_{\lambda^{\prime}}^{0}\right)} \psi_{\lambda}+\psi_{\lambda^{\prime}}, \\
& \psi_{\lambda \lambda^{\prime}}^{+} \approx \psi_{\lambda}-\frac{\left|I_{\lambda \lambda^{\prime}}\right|}{\left(\varepsilon_{\lambda}^{0}-\varepsilon_{\lambda^{\prime}}^{0}\right)} \psi_{\lambda^{\prime}},
\end{aligned}
$$

$\varepsilon_{\lambda \lambda^{\prime}}^{-} \approx \varepsilon_{\lambda^{\prime}}^{0}, \varepsilon_{\lambda \lambda^{\prime}}^{+} \approx \varepsilon_{\lambda}^{0}$. Электрон оказывается локализованным около одного из центров $\lambda$ или $\lambda^{\prime}$; при этом его волновая функция близка к атомной $\psi_{\lambda \lambda^{\prime}}^{-} \approx \psi_{\lambda^{\prime}}$, $\psi_{\lambda \lambda^{\prime}}^{+} \approx \psi_{\lambda}$, поскольку весовой множитель $\frac{\left|I_{\lambda \lambda^{\prime}}\right|}{\left(\varepsilon_{\lambda}^{0}-\varepsilon_{\lambda^{\prime}}^{0}\right)}$ за счет резонансного интеграла $I_{\lambda \lambda^{\prime}}$ экспоненциально убывает с увеличением расстояния между центрами локализации $\lambda, \lambda^{\prime}$.

С учетом (5a), (5б) для водородоподобных примесных центров (мелких локальных уровней) матричные эле- 
менты равны

$$
\begin{aligned}
\left\langle\psi_{\lambda \lambda^{\prime}}^{-}|(\mathbf{n}, \mathbf{r})| \psi_{\lambda \lambda^{\prime}}^{+}\right\rangle= & \left(\mathbf{n}, \mathbf{r}_{\lambda \lambda^{\prime}}\right) \frac{I_{\lambda \lambda^{\prime}}}{\Gamma_{\lambda \lambda^{\prime}}} \\
& +\frac{\left(\varepsilon_{\lambda}^{0}-\varepsilon_{\lambda^{\prime}}^{0}\right)\left\langle\psi_{\lambda}|(\mathbf{n}, \mathbf{r})| \psi_{\lambda^{\prime}}\right\rangle}{\Gamma_{\lambda \lambda^{\prime}}}
\end{aligned}
$$

Здесь $\left\langle\psi_{\lambda}|\mathbf{r}| \psi_{\lambda}\right\rangle=\mathbf{r}_{\lambda},\left\langle\psi_{\lambda^{\prime}}|\mathbf{r}| \psi_{\lambda^{\prime}}\right\rangle=\mathbf{r}_{\lambda^{\prime}}=\mathbf{r}_{\lambda}+\mathbf{r}_{\lambda \lambda^{\prime}}, \mathbf{r}_{\lambda}-$ радиус-вектор центра $\lambda, \mathbf{r}_{\lambda \lambda^{\prime}}-$ радиус-вектор центра $\lambda^{\prime} \quad$ относительно центра $\lambda, \quad\left\langle\psi_{\lambda}|(\mathbf{n}, \mathbf{r})| \psi_{\lambda^{\prime}}\right\rangle=$ $=\left\langle\psi_{\lambda^{\prime}}|(\mathbf{n}, \mathbf{r})| \psi_{\lambda}\right\rangle$.

Для резонансных пар уровней с затравочными энергиями $\varepsilon_{\lambda}^{0} \approx \varepsilon_{\lambda^{\prime}}^{0}$, вносящих основной вклад в бесфононную проводимость, матричные элементы (7a) принимают вид

$$
\left\langle\psi_{\lambda \lambda^{\prime}}^{-}|(\mathbf{n}, \mathbf{r})| \psi_{\lambda \lambda^{\prime}}^{+}\right\rangle \approx\left(\mathbf{n}, \mathbf{r}_{\lambda \lambda^{\prime}}\right) \frac{I_{\lambda \lambda^{\prime}}}{\Gamma_{\lambda \lambda^{\prime}}}
$$

Такие выражения используют для расчета частотной зависимости низкотемпературной бесфононной проводимости $\sigma_{1}^{\text {res }}(\omega)[6,7]$. Следует отметить, что резонансные пары уровней с затравочными энергиями $\varepsilon_{\lambda}^{0} \approx \varepsilon_{\lambda^{\prime}}^{0}$ вносят основной вклад в бесфононную проводимость при частотах $\omega<\omega_{\text {opt }} \sim \omega_{c r}$, отвечающих $r_{\omega}>r_{\text {opt. }}$ Соответственно для расчета частотной зависимости проводимости $\sigma_{1}(\omega)$ в окрестности частоты кроссовера $\omega_{c r}$ следует использовать выражение (7a).

Учет кулоновского взаимодействия между электронами, локализованными на рассматриваемой паре центров, с учетом закона сохранения энергии $\varepsilon_{\lambda \lambda^{\prime}}^{+}=\varepsilon_{\lambda \lambda^{\prime}}^{-}+\hbar \omega$ при низких температурах приводит к тому, что разность средних чисел заполнения отлична от нуля и равна единице $n_{F}\left(\varepsilon_{\lambda \lambda^{\prime}}^{-}\right)-n_{F}\left(\varepsilon_{\lambda \lambda^{\prime}}^{+}\right)=1$ при

$$
\mu-\hbar \omega-\frac{e^{2}}{\kappa r_{\lambda \lambda^{\prime}}}<\varepsilon_{\lambda \lambda^{\prime}}^{-}<\mu .
$$

С учетом (7a) выражение для проводимости (3) принимает вид

$$
\sigma_{1}^{\mathrm{res}}(\omega)=\sigma_{1 a}^{\mathrm{res}}(\omega)+\sigma_{1 b}^{\mathrm{res}}(\omega)+\sigma_{1 c}^{\mathrm{res}}(\omega)
$$

где

$$
\begin{aligned}
\sigma_{1 a}^{\mathrm{res}}(\omega)= & \frac{\pi e^{2} \omega}{V_{0}} \sum_{\substack{\left\{\lambda, \lambda^{\prime}\right\} \\
\lambda \neq \lambda^{\prime}}}\left[\frac{\left(\mathbf{n}, \mathbf{r}_{\lambda \lambda^{\prime}}\right) I_{\lambda \lambda^{\prime}}}{\Gamma_{\lambda \lambda^{\prime}}}\right]^{2} \delta\left(\varepsilon_{\lambda \lambda^{\prime}}^{-}-\varepsilon_{\lambda \lambda^{\prime}}^{+}+\hbar \omega\right), \\
\sigma_{1 b}^{\mathrm{res}}(\omega)= & \frac{\pi e^{2} \omega}{V_{0}} \sum_{\substack{\left\{\lambda, \lambda^{\prime}\right\} \\
\lambda \neq \lambda^{\prime}}} 2 \frac{\left(\mathbf{n}, \mathbf{r}_{\lambda \lambda^{\prime}}\right) I_{\lambda \lambda^{\prime}}}{\Gamma_{\lambda \lambda^{\prime}}} \\
& \times \frac{\left(\varepsilon_{\lambda}^{0}-\varepsilon_{\lambda^{\prime}}^{0}\right)\left\langle\psi_{\lambda}|(\mathbf{n}, \mathbf{r})| \psi_{\lambda^{\prime}}\right\rangle}{\Gamma_{\lambda \lambda^{\prime}}} \delta\left(\varepsilon_{\lambda \lambda^{\prime}}^{-}-\varepsilon_{\lambda \lambda^{\prime}}^{+}+\hbar \omega\right), \\
\sigma_{1 c}^{\mathrm{res}}(\omega)= & \frac{\pi e^{2} \omega}{V_{0}} \sum_{\substack{\left\{\lambda, \lambda^{\prime}\right\} \\
\lambda \neq \lambda^{\prime}}}\left[\frac{\left(\varepsilon_{\lambda}^{0}-\varepsilon_{\lambda^{\prime}}^{0}\right)\left\langle\psi_{\lambda}|(\mathbf{n}, \mathbf{r})| \psi_{\lambda^{\prime}}\right\rangle}{\Gamma_{\lambda \lambda^{\prime}}}\right]^{2} \\
& \times \delta\left(\varepsilon_{\lambda \lambda^{\prime}}^{-}-\varepsilon_{\lambda \lambda^{\prime}}^{+}+\hbar \omega\right) .
\end{aligned}
$$

Как известно, для расчета прыжковой проводимости важно знать асимптотику волновых функций локализованных носителей заряда. В случае, когда расстояние между центрами в паре больше радиуса локализации, $r_{\lambda, \lambda^{\prime}}>a$, величины матричных элементов можно непосредственно оценить. Для водородоподобных примесей в приближении изотропного закона дисперсии выражение для матричного элемента имеет вид [11]

$$
\begin{aligned}
\left\langle\lambda|(\mathbf{n}, \mathbf{r})| \lambda^{\prime}\right\rangle & =\left\langle\psi_{\lambda}|(\mathbf{n}, \mathbf{r})| \psi_{\lambda^{\prime}}\right\rangle \\
& \approx \frac{r_{\lambda \lambda^{\prime}}^{3}}{a^{2}} \exp \left(-r_{\lambda \lambda^{\prime}} / a\right) \cos \theta
\end{aligned}
$$

где $\theta-$ угол между векторами $\mathbf{n}$ и $\mathbf{r}_{\lambda \lambda^{\prime}}, \psi_{\lambda}=\left(1 / \sqrt{\pi a^{3}}\right)$ $\times \exp \left(-\left|\mathbf{r}-\mathbf{r}_{\lambda}\right| / a\right)$. Действительно, при большом межцентровом расстоянии в паре $r_{\lambda, \lambda^{\prime}} \gg a$ основной вклад в матричный элемент $\left\langle\lambda|(\mathbf{n}, \mathbf{r})| \lambda^{\prime}\right\rangle$ дает интегрирование по сигарообразной области, вытянутой вдоль отрезка $r_{\lambda, \lambda^{\prime}}$, внутри которой произведение волновых функций $\psi_{\lambda}, \psi_{\lambda^{\prime}}$ можно считать постоянным и равным

$$
\psi_{\lambda} \psi_{\lambda^{\prime}} \approx\left(1 / a^{3}\right) \exp \left(-r_{\lambda, \lambda^{\prime}} / a\right)
$$

Граница области, интегрирование по которой дает основной вклад в матричный элемент $\left\langle\lambda|(\mathbf{n}, \mathbf{r})| \lambda^{\prime}\right\rangle$ представляет собой поверхность, на которой значение произведения

$$
\psi_{\lambda} \psi_{\lambda^{\prime}} \approx\left(1 / a^{3}\right) \exp \left(-r_{\lambda, \lambda^{\prime}} / a\right) \exp \left(-\frac{1}{2} \frac{r_{\perp}^{2} r_{\lambda, \lambda^{\prime}}}{a\left(r_{\lambda, \lambda^{\prime}}-r_{\|}\right) r_{\|}}\right)
$$

уменьшается в $e$ раз по сравнению со значением на оси (10a); площадь поперечного сечения области порядка $S(\xi) \sim r_{\perp}^{2} \approx 2 a r_{\lambda, \lambda^{\prime}} \xi(1-\xi)$, где $\xi=r_{\|} / r_{\lambda, \lambda^{\prime}}$, $\mathbf{r}_{\perp}, \mathbf{r}_{\|}$- перпендикулярная и параллельная отрезку $r_{\lambda, \lambda^{\prime}}$ составляющие вектора $\mathbf{r}=\mathbf{r}_{\perp}+\mathbf{r}_{\|}, \xi \in[0,1]$ (начало отсчета совпадает с одним из центров, например, $\lambda$ ). Оценка матричного элемента дает

$$
\begin{aligned}
\left\langle\lambda|(\mathbf{n}, \mathbf{r})| \lambda^{\prime}\right\rangle & =\int_{V} \psi_{\lambda^{\prime}}(\mathbf{n}, \mathbf{r}) \psi_{\lambda} d \mathbf{r} \approx \psi_{\lambda^{\prime}} \psi_{\lambda} \int_{V}(\mathbf{n}, \mathbf{r}) d \mathbf{r} \\
& \approx \psi_{\lambda^{\prime}} \psi_{\lambda} \cos \theta r_{\lambda \lambda^{\prime}}^{2} \int_{0}^{1} \xi S(\zeta) d \xi \\
& \approx \frac{r_{\lambda \lambda^{\prime}}^{3}}{a^{2}} \exp \left(-r_{\lambda \lambda^{\prime}} / a\right) \cos \theta
\end{aligned}
$$

Степенная зависимость предэкспоненциального множителя в выражении для матричного элемента $\left\langle\lambda|(\mathbf{n}, \mathbf{r})| \lambda^{\prime}\right\rangle$ от межцентрового расстояния $r_{\lambda, \lambda^{\prime}}$ определяется объемом сигарообразной области, увеличивающимся с ростом межцентрового расстояния. Используемый подход к оценке матричных элементов может быть применен и в случае волновых функций с другими асимптотиками. 
Матричный элемент имеет резкий максимум при $r_{\mathrm{opt}}=3 a$, поскольку с уменьшением расстояния между центрами в паре уменьшается изменение дипольного момента системы при электронном переходе, а с увеличением расстояния между центрами происходит экспоненциальное уменьшение перекрытия волновых функций состояний, отвечающих центрам локализации. В этом случае оптимальная длина прыжка $r_{\text {opt }}$ определяется параметрами системы и слабо зависит от частоты. В дальнейших вычислениях мы используем оценку (10), поскольку основной вклад в бесфононную проводимость вносят пары, расстояния между центрами в которых больше радиуса локализации $a$.

Переходя в $(9 \mathrm{a})-(9 \mathrm{~B})$ от суммирования к интегрированию, получаем

$$
\begin{aligned}
& \sigma_{1 a}^{\mathrm{res}}(\omega)=\frac{4 \pi^{2} e^{2} \rho_{0}^{2} \omega}{3} \int_{r_{\omega}}^{\infty} d r_{\lambda \lambda^{\prime}} r_{\lambda \lambda^{\prime}}^{4} \int_{\mu-\hbar \omega-\frac{e^{2}}{k r \lambda \lambda^{\prime}}}^{\mu} d \varepsilon_{\lambda \lambda^{\prime}}^{-} \int d \varepsilon_{\lambda \lambda^{\prime}}^{+} \\
& \times \Phi\left(\varepsilon_{\lambda \lambda^{\prime}}^{-}, \varepsilon_{\lambda \lambda^{\prime}}^{+}, r_{\lambda \lambda^{\prime}}\right) \frac{I^{2}\left(r_{\lambda \lambda^{\prime}}\right)}{\left(\varepsilon_{\lambda \lambda^{\prime}}^{+}-\varepsilon_{\lambda \lambda^{\prime}}^{-}\right)^{2}} \delta\left(\varepsilon_{\lambda \lambda^{\prime}}^{-}-\varepsilon_{\lambda \lambda^{\prime}}^{+}+\hbar \omega\right), \\
& \sigma_{1 b}^{\mathrm{res}}(\omega)=\frac{4 \pi^{2} e^{2} \rho_{0}^{2} \omega}{3} \int_{r_{\omega}}^{\infty} d r_{\lambda \lambda^{\prime}} \frac{r_{\lambda \lambda^{\prime}}^{6}}{a^{2}} \exp \left(-r_{\lambda \lambda^{\prime}} / a\right) \\
& \times \quad \int_{\varepsilon^{2}}^{\mu} d \varepsilon_{\lambda \lambda^{\prime}}^{-} \int d \varepsilon_{\lambda \lambda^{\prime}}^{+} \Phi\left(\varepsilon_{\lambda \lambda^{\prime}}^{-}, \varepsilon_{\lambda \lambda^{\prime}}^{+}, r_{\lambda \lambda^{\prime}}\right) \frac{2 I_{\lambda \lambda^{\prime}}}{\left(\varepsilon_{\lambda \lambda^{\prime}}^{+}-\varepsilon_{\lambda \lambda^{\prime}}^{-}\right)^{2}} \\
& \mu-\hbar \omega-\frac{e^{2}}{k r \lambda \lambda^{\prime}} \\
& \times \sqrt{\left(\varepsilon_{\lambda \lambda^{\prime}}^{+}-\varepsilon_{\lambda \lambda^{\prime}}^{-}\right)^{2}-4 I_{\lambda \lambda^{\prime}}^{2}} \delta\left(\varepsilon_{\lambda \lambda^{\prime}}^{-}-\varepsilon_{\lambda \lambda^{\prime}}^{+}+\hbar \omega\right), \\
& \sigma_{1 c}^{\mathrm{res}}(\omega)=\frac{4 \pi^{2} e^{2} \rho_{0}^{2} \omega}{3} \int_{r_{\omega}}^{\infty} d r_{\lambda \lambda^{\prime}} \frac{r_{\lambda \lambda^{\prime}}^{8}}{a^{4}} \exp \left(-2 r_{\lambda \lambda^{\prime}} / a\right) \\
& \times \int_{\mu-\hbar \omega-\frac{e^{2}}{k r_{\lambda \lambda^{\prime}}}}^{\mu} d \varepsilon_{\lambda \lambda^{\prime}}^{-} \int d \varepsilon_{\lambda \lambda^{\prime}}^{+} \Phi\left(\varepsilon_{\lambda \lambda^{\prime}}^{-}, \varepsilon_{\lambda \lambda^{\prime}}^{+}, r_{\lambda \lambda^{\prime}}\right) \\
& \times \frac{\left(\left(\varepsilon_{\lambda \lambda^{\prime}}^{+}-\varepsilon_{\lambda \lambda^{\prime}}^{-}\right)^{2}-4 I_{\lambda \lambda^{\prime}}^{2}\right)}{\left(\varepsilon_{\lambda \lambda^{\prime}}^{+}-\varepsilon_{\lambda \lambda^{\prime}}^{-}\right)^{2}} \delta\left(\varepsilon_{\lambda \lambda^{\prime}}^{-}-\varepsilon_{\lambda \lambda^{\prime}}^{+}+\hbar \omega\right),
\end{aligned}
$$

где

$$
\Phi\left(\varepsilon_{\lambda \lambda^{\prime}}^{-}, \varepsilon_{\lambda \lambda^{\prime}}^{+}, r_{\lambda \lambda^{\prime}}\right)=\frac{\varepsilon_{\lambda \lambda^{\prime}}^{+}-\varepsilon_{\lambda \lambda^{\prime}}^{-}}{\sqrt{\left(\varepsilon_{\lambda \lambda^{\prime}}^{+}-\varepsilon_{\lambda \lambda^{\prime}}^{-}\right)^{2}-4 I_{\lambda \lambda^{\prime}}^{2}}}
$$

есть якобиан перехода от затравочных энергий $\varepsilon_{\lambda}^{0}, \varepsilon_{\lambda^{\prime}}^{0}$ (без учета гибридизации) к энергиям $\varepsilon_{\lambda \lambda^{\prime}}^{-}, \varepsilon_{\lambda \lambda^{\prime}}^{+}$;

$$
\Gamma_{\lambda \lambda^{\prime}}=\varepsilon_{\lambda \lambda^{\prime}}^{+}-\varepsilon_{\lambda \lambda^{\prime}}^{-}=\sqrt{\left(\varepsilon_{\lambda}^{0}-\varepsilon_{\lambda^{\prime}}^{0}\right)^{2}+4 I_{\lambda \lambda^{\prime}}^{2}} .
$$

Проинтегрировав (11a)-(11в) по энергиям $\varepsilon_{\lambda \lambda^{\prime}}^{-}, \varepsilon_{\lambda \lambda^{\prime}}^{+}$, получаем

$$
\begin{aligned}
& \sigma_{1 a}^{\mathrm{res}}(\omega)=\frac{4 \pi^{2} e^{2} \rho_{0}^{2}}{3 \hbar^{2}} \\
& \times \int_{r_{\omega}}^{\infty} r_{\lambda \lambda^{\prime}}^{4}\left(\hbar \omega+\frac{e^{2}}{\kappa r_{\lambda \lambda^{\prime}}}\right) \frac{I_{\lambda \lambda^{\prime}}^{2}}{\sqrt{(\hbar \omega)^{2}-4 I_{\lambda \lambda^{\prime}}^{2}}} d r_{\lambda \lambda^{\prime}} \\
& \sigma_{1 b}^{\mathrm{res}}(\omega)=\frac{4 \pi^{2} e^{2} \rho_{0}^{2} \omega_{c}}{3} \\
& \times \int_{r_{\omega}}^{\infty} \frac{r_{\lambda \lambda^{\prime}}^{6}}{a^{2}}\left(\hbar \omega+\frac{e^{2}}{\kappa r_{\lambda \lambda^{\prime}}}\right) \exp \left(-2 r_{\lambda \lambda^{\prime}} / a\right) d r_{\lambda \lambda^{\prime}}, \\
& \sigma_{1 c}^{\mathrm{res}}(\omega)=\frac{4 \pi^{2} e^{2} \rho_{0}^{2}}{3 \hbar} \int_{r_{\omega}}^{\infty} \frac{r_{\lambda \lambda^{\prime}}^{8}}{a^{4}}\left(\hbar \omega+\frac{e^{2}}{\kappa r_{\lambda \lambda^{\prime}}}\right) \\
& \quad \times \exp \left(-2 r_{\lambda \lambda^{\prime}} / a\right) \sqrt{(\hbar \omega)^{2}-4 I_{\lambda \lambda^{\prime}}^{2}} d r_{\lambda \lambda^{\prime}}
\end{aligned}
$$

где

$$
\begin{gathered}
I_{\lambda \lambda^{\prime}}=I_{0} \exp \left(-r_{\lambda \lambda^{\prime}} / a\right), \quad I_{0} \approx e^{2} / \kappa a, \\
\omega_{c}=2 I_{0} / \hbar, \quad \hbar \omega=2 I_{\lambda \lambda^{\prime}}\left(r_{\omega}\right) .
\end{gathered}
$$

В области частот $\omega \ll \omega_{c}$, с учетом равенства

$$
\int_{r_{\omega}}^{\infty} \frac{I_{\lambda \lambda^{\prime}}^{2}}{\sqrt{(\hbar \omega)^{2}-4 I_{\lambda \lambda^{\prime}}^{2}}} d r_{\lambda \lambda^{\prime}}=\frac{a \hbar \omega}{4}
$$

из (12a) следует известное выражение для бесфононной проводимости $[6,14]$

$$
\sigma_{1 a}^{\mathrm{res}}(\omega)=\frac{\pi^{2} e^{2} a \rho_{0}^{2}}{3} r_{\omega}^{4} \omega\left(\hbar \omega+\frac{e^{2}}{\kappa r_{\omega}}\right)
$$

При частотах $\omega<\omega_{\mathrm{opt}}$, отвечающих $r_{\omega}>r_{\mathrm{opt}}$ (в режиме проводимости с переменной длиной прыжка $\left.r_{\omega}\right)$, вклады от слагаемых (12б), (12в) в бесфононную проводимость (8) экспоненциально малы, поскольку подынтегральные выражения в (12б), (12в) имеют резкий максимум в окрестности $r_{\text {opt. }}$. Соответственно при частотах $\omega<\omega_{\text {opt }} \sim \omega_{c r}$ основной вклад в бесфононную проводимость вносят резонансные пары уровней с затравочными энергиями $\varepsilon_{\lambda}^{0} \approx \varepsilon_{\lambda^{\prime}}^{0}$.

При высоких частотах $\omega>\omega_{\text {opt }} \sim \omega_{c r}$, отвечающих $r_{\omega}<r_{\mathrm{opt}}$ в режиме с постоянной длиной прыжка $r_{\text {opt }}$, определяющий вклад в бесфононную проводимость (8) вносит слагаемое (12в), которое можно пред- 
ставить в виде

$$
\begin{aligned}
\sigma_{1 c}^{\mathrm{res}}(\omega) & \approx \frac{4 \pi^{2} e^{2} \rho_{0}^{2} \omega}{3} \\
& \times \int_{0}^{\infty} \frac{r_{\lambda \lambda^{\prime}}^{8}}{a^{4}}\left(\hbar \omega+\frac{e^{2}}{\kappa r_{\lambda \lambda^{\prime}}}\right) \exp \left(-2 r_{\lambda \lambda^{\prime}} / a\right) d r_{\lambda \lambda^{\prime}}
\end{aligned}
$$

Здесь $\sqrt{(\hbar \omega)^{2}-4 I_{\lambda \lambda^{\prime}}^{2}\left(r_{\lambda \lambda^{\prime}}\right)} \approx \hbar \omega$ при $r_{\omega}+a<r_{\lambda \lambda^{\prime}}$. Интегрирование (14) дает для бесфононной проводимости выражение (2).

Соотношения (13), (12б), (12в), (2) удобно представить в виде

$$
\begin{aligned}
& \sigma_{1 a}^{\mathrm{res}}(\omega)=\sigma_{0}\left(\ln \left(\frac{\omega_{c}}{\omega}\right)\right)^{4} \frac{\omega}{\omega_{c}}\left(A^{-1} \frac{\omega}{\omega_{c}}+\frac{1}{\ln \left(\frac{\omega_{c}}{\omega}\right)}\right) . \\
& \sigma_{1 b}^{\mathrm{res}}(\omega)=C_{0} \sigma_{0} \int_{2 \ln \left(\omega_{c} / \omega\right)}^{\infty} x^{6}\left(\frac{\omega}{\omega_{c}}+\frac{1}{x}\right) \exp (-x) d x, \\
& \sigma_{1 c}^{\mathrm{res}}(\omega)=C_{2} \sigma_{0} \frac{\omega}{\omega_{c}} \\
& \times \int_{2 \ln \left(\omega_{c} / \omega\right)}^{\infty} x^{8}\left(\frac{\omega}{\omega_{c}}+\frac{1}{x}\right) \exp (-x) \sqrt{1-\frac{\exp (-x)}{\left(\omega / \omega_{c}\right)^{2}}} d x, \\
& \sigma_{1}^{\mathrm{res}}(\omega)=C_{1} \sigma_{0} \frac{\omega}{\omega_{c}}\left(A^{-1} \frac{\omega}{\omega_{c}}+C_{3}\right),
\end{aligned}
$$

где

$$
\begin{gathered}
\sigma_{0}=\frac{\pi^{2} e^{4} \rho_{0}^{2} a^{4} \omega_{c}}{3 \kappa}, \quad \omega_{c}=2 I_{0} / \hbar, \quad I_{0}=e^{2} / \kappa a, \\
A=\frac{e^{2}}{\kappa a \hbar \omega_{c}}=\frac{e^{2}}{2 I_{0} \kappa a} \approx \frac{1}{2}, \quad x=2 r_{\lambda \lambda^{\prime}} / a \\
C_{0}=1 / 2^{4}, \quad C_{2}=1 / 2^{6}, \quad C_{3}=1 / 4
\end{gathered}
$$

— численные коэффициенты.

\section{3. Заключение}

На рис. 1, а приведена зависимость бесфононной проводимости $\sigma_{1}^{\mathrm{res}}\left(\omega / \omega_{c}\right)$ (8) от частоты в области кроссовера от линейной зависимости к квадратичной; также на рис. 1, $a$ приведены частотные зависимости отдельных слагаемых (15a)-(15в). Проводимость нормирована на величину $\sigma_{0}=\pi^{2} e^{4} a^{4} \rho_{0}^{2} \omega_{c} / 3 \kappa$; для случая $\mathrm{Si}: \mathrm{P}$ при $a \sim 30 \AA, \rho_{0} \sim 10^{18} \mathrm{~cm}^{-3} \cdot \mathrm{B}^{-1}, \kappa \approx 12$, $\omega_{c} \sim 10^{13}$ рад $\cdot \mathrm{c}^{-1}$ имеем $\sigma_{0} \approx 0.007 \mathrm{OM}^{-1} \cdot \mathrm{cm}^{-1}$. Для сравнения на рис. $1, b$ приведена частотная зависимость бесфононного вклада в проводимость в режиме проводимости с постоянной длиной прыжка (кривая 2). Расчет $\sigma_{1}^{\text {res }}\left(\omega / \omega_{c}\right)$ показал (рис. $\left.1, a, b\right)$, что при частотах
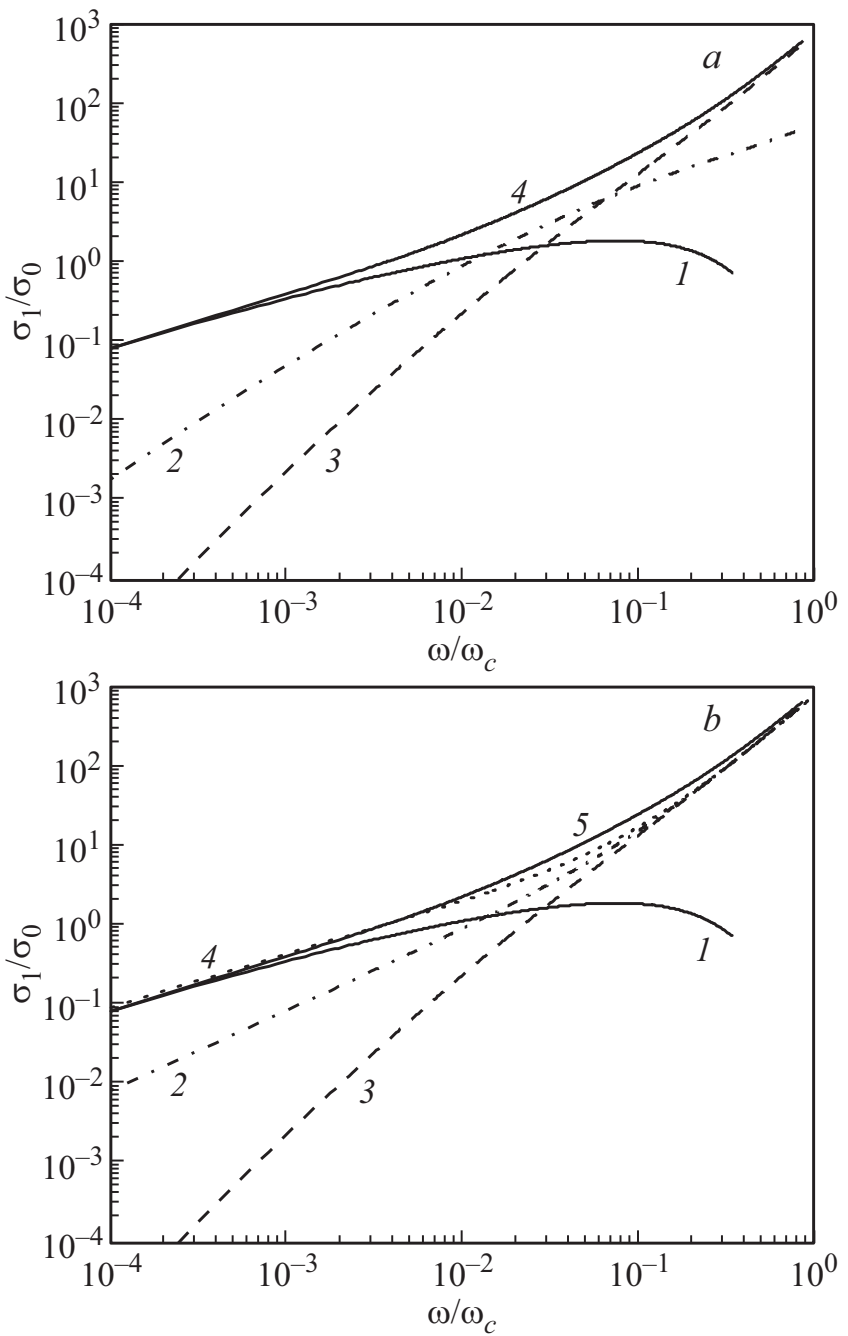

Рис. 1. $а$ - частотные зависимости слагаемых вещественной части проводимости $\sigma_{1}^{\text {res }} / \sigma_{0}: 1-(15 \mathrm{a}), 2-(156)$, $3-(15 \mathrm{~B}) ; 4-$ частотная зависимость бесфононной проводимости $\sigma_{1}^{\text {res }} / \sigma_{0}(8) . b-$ частотные зависимости слагаемых вещественной части проводимости $\sigma_{1}^{\text {res }} / \sigma_{0}: 1-(15 \mathrm{a}), 3-(15 \mathrm{~B})$; 2 - частотная зависимость бесфононной проводимости $\sigma_{1}^{\text {res }} / \sigma_{0}$ в режиме проводимости с постоянной длиной прыжка (2), (14); 4 - экстраполяция модели с переменной длиной прыжка (1) на область квадратичной частотной зависимости в режиме проводимости с постоянной длиной прыжка $(2) ; 5$ - частотная зависимость бесфононной проводимости $\sigma_{1}^{\text {res }} / \sigma_{0}(8)$.

$\omega<\omega_{\text {opt }} \sim \omega_{c r}$ (в режиме с переменной длиной прыжка) бесфононная проводимость определяется выражением (13). При высоких частотах $\omega>\omega_{\text {орt }}$ (в режиме с постоянной длиной прыжка) выражение для бесфононной проводимости принимает вид (2), (14); в этом случае, определяющий вклад в бесфононную проводимость (8) вносит слагаемое (12в). Согласно рис. $1, b$, используемая в [11] для анализа поведения частотной характеристики экстраполяция модели с переменной длиной прыжка на область квадратичной частотной зависимости в режиме проводимости с постоянной длиной прыжка согласуется 


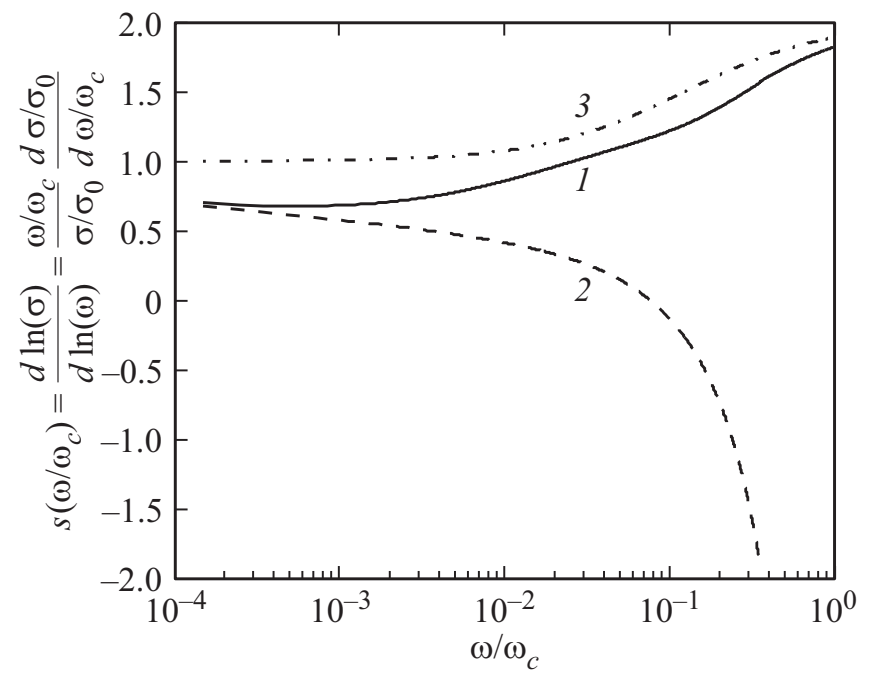

Рис. 2. Частотные зависимости $s\left(\omega / \omega_{c}\right)=\frac{d \ln (\sigma)}{d \ln (\omega)}=\frac{\omega / \omega_{c}}{\sigma / \sigma_{0}}$ $\times \frac{d \sigma / \sigma_{0}}{d \omega / \omega_{c}}$. Кривая 1 соответствует бесфононной проводимости (8); кривая 2 соответствует бесфононной проводимости в режиме с переменной длиной прыжка (15a); кривая 3 соответствует бесфононной проводимости в режиме с постоянной длиной прыжка (2), (14). Кривая 3 отвечает режиму проводимости с постоянной длиной прыжка $r_{\text {орt }}$ во всей области перехода от линейной к квадратичной частотной зависимости проводимости.

с результатами расчета частотной зависимости проводимости в области перехода от линейной зависимости к квадратичной.

На рис. 2 видно, что частотная зависимость бесфононной проводимости (8) в низкочастотной области $\omega<\omega_{c r}$ (при $\left.\hbar \omega<e^{2} / \kappa r_{\omega}\right)$ имеет сублинейный характер $\left(\sigma_{1}^{\text {res }}(\omega) \sim \omega^{s}\right.$, где $\left.s \approx 0.8\right)$. В области высоких частот, когда эффекты, связанные с гибридизацией локализованных состояний, становятся несущественны, происходит переход к режиму проводимости с постоянной длиной прыжка (2), (14), когда основной вклад в проводимость вносят электронные переходы внутри пар с оптимальными межцентровыми расстояниями $r_{\text {орt }} \approx 3 a$, слабо зависящими от частоты. Согласно вычислениям переход к режиму проводимости с постоянной длиной прыжка $r_{\text {opt }}$ происходит при $\omega \sim \omega_{\text {opt }} \approx 0.05 \omega_{c}$, когда $r_{\mathrm{opt}} \sim r_{\omega}$; в этом случае при $\omega>\omega_{\mathrm{opt}}\left(r_{\mathrm{opt}}>r_{\omega}\right)$ основной вклад в проводимость вносят электронные переходы внутри пар с межцентровыми расстояниями $r_{\lambda \lambda^{\prime}} \sim r_{\text {opt. }}$. Отметим, что частота $\omega_{\text {opt }} \approx 0.05 \omega_{c}$, при которой происходит указанный выше переход, порядка частоты кроссовера в (2) $\hbar \omega_{c r} \approx e^{2} / \kappa r_{\text {opt }}$, т.е. $\omega_{c r} \approx 0.1 \omega_{c}$. Соответственно переход от линейной к квадратичной частотной зависимости вещественной части проводимости $\sigma_{1}(\omega)$ осуществляется в окрестности частоты $\omega \sim \omega_{\text {орt}}$, которая отвечает $r_{\omega} \sim r_{\text {орt. }}$ Частота $\omega_{\text {opt }}$, при которой происходит переход к режиму с постоянной длиной прыжка $r_{\text {opt }}$, соответствует положению максимума $\sigma_{1}^{\text {res }}(\omega)$ в стандартной теории бесфононной проводимости $\omega_{m} \approx 0.07 \omega_{c}$. Немонотонность $\sigma_{1}^{\text {res }}(\omega)$ в режиме проводимости с переменной длиной прыжка $r_{\omega}$ обусловлена частотной зависимостью оптимальной длины прыжка $r_{\omega}$.

Таким образом, проведенный в работе расчет показал, что изменение режима проводимости (переход от переменной к постоянной длине прыжка с ростом частоты) обусловливает переход от линейной к квадратичной частотной зависимости бесфононной проводимости в терагерцовом диапазоне частот в окрестности $\omega_{c r}$.

\section{Конфликт интересов}

Авторы заявляют, что у них нет конфликта интересов.

\section{Список литературы}

[1] I.P. Zvyagin. In: Charge Transport in Disordered Solids with Applications in Electronics, ed. by S. Baranovski (John Wiley \& Sons, Chichester, 2006) p. 339.

[2] M. Pollak, M. Ortuño, A. Frydman. The Electron Glass (Cambridge University Press, 2013).

[3] M. Pollak, T.H. Geballe. Phys. Rev., 122, 1742 (1961).

[4] I.G. Austin, N.F. Mott. Adv. Phys., 18, 41 (1969).

[5] A.L. Efros. Phil. Mag. B, 43, 829 (1981).

[6] Б.И. Шкловский, А.Л. Эфрос. ЖЭТФ, 81, 406 (1981).

[7] N.F. Mott. Phil. Mag., 22, 7 (1970).

[8] E. Helgren, N.P. Armitage, G. Gruner. Phys. Rev. B, 69, 014201 (2004).

[9] M. Hering, M. Scheffler, M. Dressel, H.v. Lohneysen. Phys. Rev. B, 75, 205203 (2007).

[10] И.П. Звягин, М.А. Ормонт. Вестн. Моск. ун-та. Физика, Астрономия, № 4, 44 (2008).

[11] М.А. Ормонт, И.П. Звягин. ФТП, 49 (4), 449 (2015).

[12] М.А. Ормонт, И.П. Звягин. ФТП, 52 (2), 161 (2018).

[13] В.Л. Бонч-Бруевич, И.П. Звягин, Р. Кайпер, А.Г. Миронов, Р. Эндерлайн, Б. Эссер. Электронная теория неупорядоченных полупроводников (М., Наука, 1981).

[14] A.L. Efros, B.I. Shklovskii. In: Electron-Electron Interactions in Disordered Systems, ed. by A.L. Efros, M. Pollak (NorthHolland, Elsevier Science Publishers B.V., Amsterdam, 1985) p. 409.

Редактор Г.А. Оганесян 
High-frequency conductivity of disordered semiconductors in the transition region from linear to quadratic frequency dependence

M.A. Ormont, I.P. Zvyagin

Moscow State University (Faculty of Physics), 119991 Moscow, Russia

Abstract The features of the high-frequency conductivity of disordered semiconductors associated with the hopping electron transport through the impurity band are considered. In the pair approximation, we calculated the frequency dependence of the real part of low- temperature phononless conductivity in the transition region (crossover) from linear to quadratic frequency dependence of conductivity. It is shown that the crossover in the terahertz frequency range is associated with a transition from the variablerange hopping regime to the fixed-range hopping conductivity regime with increasing frequency. 